\title{
Efficient computation of trees with minimal atom-bond connectivity index
}

\author{
Darko Dimitrov \\ Institut für Informatik, Freie Universität Berlin, \\ Takustraße 9, D-14195 Berlin, Germany \\ E-mail: darko@mi.fu-berlin.de
}

\begin{abstract}
The atom-bond connectivity $(A B C)$ index is one of the recently most investigated degree-based molecular structure descriptors, that have applications in chemistry. For a graph $G$, the ABC index is defined as $\sum_{u v \in E(G)} \sqrt{\frac{(d(u)+d(v)-2)}{d(u) d(v)}}$, where $d(u)$ is the degree of vertex $u$ in $G$ and $E(G)$ is the set of edges of $G$. Despite many attempts in the last few years, it is still an open problem to characterize trees with minimal $A B C$ index. In this paper, we present an efficient approach of computing trees with minimal ABC index, by considering the degree sequences of trees and some known properties of trees with minimal $A B C$ index. The obtained results disprove some existing conjectures and suggest new ones to be set.
\end{abstract}

\section{Introduction and some related results}

Molecular descriptors [44] are mathematical quantities that describe the structure or shape of molecules, helping to predict the activity and properties of molecules in complex experiments. Among them, so-called topological indices [18] play a significant role. The topological indices can be classified by the structural properties of graphs used for their calculation. For example, the Wiener index [47] and the Balaban $J$ index [5] are based on the distance of vertices in the respective graph, the Estrada index [19] and the energy of a graph [29] are based on the spectrum of the graph, the Zagreb group indices [34] and the Randic connectivity index [40] depend on the degrees of vertices, while the Hosoya index [35] is calculated by counting of non-incident edges in a graph. On the other hand, there is a group of so-called information indices that are based on information functionals [6]. More about the information indices and the discriminative power of some established indices, one can find in $[15,16,17,24]$ and in the works cited therein.

Here, we consider a relatively new topological index which attracted a lot of attention last few years. Namely, in 1998, Estrada et al. [21] proposed a new vertex-degree-based graph topological index, the atom-bond connectivity $(A B C)$ index, and showed that it can 
be a valuable predictive tool in the study of the heat of formation in alikeness. Ten years later Estrada [20] elaborated a novel quantum-theory-like justification for this topological index. After that revelation, the interest of $\mathrm{ABC}$-index has grown rapidly. Additionaly, the physico-chemical applicability of the $\mathrm{ABC}$ index was confirmed and extended in several studies $[3,10,14,28,33,38,51]$.

Let $G=(V, E)$ be a simple undirected graph of order $n=|V|$ and size $m=|E|$. For $v \in V(G)$, the degree of $v$, denoted by $d(v)$, is the number of edges incident to $v$. Then the atom-bond connectivity index of $G$ is defined as

$$
\operatorname{ABC}(G)=\sum_{u v \in E(G)} \sqrt{\frac{(d(u)+d(v)-2)}{d(u) d(v)}} .
$$

As a new and well motivated graph invariant, the $\mathrm{ABC}$ index has attracted a lot of interest in the last several years both in mathematical and chemical research communities and numerous results and structural properties of $\mathrm{ABC}$ index were established $[7,8,9,11$, $12,13,22,23,26,30,32,39,45,48,49,50]$.

The fact that adding an edge in a graph strictly increases its $\mathrm{ABC}$ index [12] (or equivalently that deleting an edge in a graph strictly decreases its $\mathrm{ABC}$ index [7]) has the following two immediate consequences.

Corollary 1.1. Among all connected graphs with $n$ vertices, the complete graph $K_{n}$ has maximal value of $A B C$ index.

Corollary 1.2. Among all connected graphs with $n$ vertices, the graph with minimal $A B C$ index is a tree.

Although it is fairly easy to show that the star graph $S_{n}$ is a tree with maximal ABC index [23], despite many attempts in the last years, it is still an open problem the characterization of trees with minimal ABC index (also refereed as minimal-ABC trees). To accomplish that task, besides the theoretically proven properties of the trees with minimal ABC index, computer supported search can be of enormous help. A good example of that is the work done by Furtula et al. [25], where the trees with minimal ABC index of up to size of 31 were computed. There, a brute-force approach of generating all trees of a given order, speeded up by using a distributed computing platform, was applied.

Here, we improve the computer search in two ways. Firstly, we consider only the degree sequences of trees. We would like to stress that the number of degree sequences of a given length $n$ is significantly smaller than the number of all trees of order $n$. For example, the number of trees with 32 vertices is 109972410221 [43], while the number of degree sequences of length 32 is 5604 (see Table 1). Secondly, to speed up the computation, we generate only degree sequences of trees that correspond to some known structural properties of the trees with minimal ABC index (Propositions 3.1, 3.2 and 3.3 from Section 3 ). Thus, using a single PC, we have identified all trees with minimal ABC of order up to 300. The obtained results strengthen the believe that some conjectures are true and reject other conjectures.

In the sequel, we present some additional results and notation that will be used in the rest of the paper. A vertex of degree one is a pendant vertex. As in [32], a sequence of vertices of a graph $G, S_{k}=v_{0} v_{1} \ldots v_{k}$, will be called a pendant path if each two consecutive vertices in 
$S_{k}$ are adjacent in $G, d\left(v_{0}\right)>2, d\left(v_{i}\right)=2$, for $i=1, \ldots, k-1$, and $d\left(v_{k}\right)=1$. The length of the pendant path $S_{k}$ is $k$. A sequence $D=\left(d_{1}, d_{2}, \ldots, d_{n}\right)$ is graphical if there is a graph whose vertex degrees are $d_{i}, i=1, \ldots, n$. If in addition $d_{1} \geq d_{2} \geq \cdots \geq d_{n}$, then $D$ is a degree sequence. Let $\mathbf{D}_{\mathbf{n}}$ be the set of all degree sequences of trees of length $n$.

In [46] Wang defined a greedy tree as follows.

Definition $1.1([46])$. Suppose the degrees of the non-leaf vertices are given, the greedy tree is achieved by the following 'greedy algorithm':

1. Label the vertex with the largest degree as $v$ (the root).

2. Label the neighbors of $v$ as $v_{1}, v_{2}, \ldots$, assign the largest degree available to them such that $d\left(v_{1}\right) \geq d\left(v_{2}\right) \geq \ldots$

3. Label the neighbors of $v_{1}$ (except $v$ ) as $v_{11}, v_{12}, \ldots$ such that they take all the largest degrees available and that $d\left(v_{11}\right) \geq d\left(v_{12}\right) \geq \ldots$ then do the same for $v_{2}, v_{3}, \ldots$

4. Repeat 3. for all newly labeled vertices, always starting with the neighbors of the labeled vertex with largest whose neighbors are not labeled yet.

The following result by Gan, Liu and You [27] characterizes the trees with minimal ABC index with prescribed degree sequences.

Theorem 1.3. Given the degree sequence, the greedy tree minimizes the $A B C$ index.

The same result as in Theorem 1.3, using slightly different notation and approach, was obtained by Xing and Zhou [48]. Since the Theorem 1.3 plays a crucial role in our computation, the first important issue is how to enumerate efficiently degree sequences of trees. This problem is considered in the next section.

\section{Enumerating degree sequences of trees}

There exist several algorithms for enumerating degree sequences of graphs. A comprehensive source of references of such algorithms can be found in [37]. Clearly, each of those algorithms can be used for enumerating degree sequences of trees just by considering only the degree sequences with sum of degrees equals to $2 n-2$, where $n$ is the length of the degree sequences. However, this is not an efficient approach, because most of the generated degree sequences are not degree sequences of trees. For an illustration, the number of all degree sequences of length 29 is 2022337118015338 [42], while the number of degree sequences that correspond to trees of order 29 is 3010 (see Table 1). Thus, it is not a surprise that the largest reported enumerated degree sequences of graphs was only of length 29, with running time of 6733 days, distributed to 200 PCs containing about 700 cores [37]. Since we are not aware of an algorithm specialized only for enumerating degree sequences of trees, we present such an algorithm in this section. Our algorithm is related to the algorithm of enumerating degree sequences of graphs presented by Ruskey et al [41], and exploit the so called "reverse search", a term originated by Avis and Fukuda [4]. Therefore, in the sequel, we will adopt the notation used in [41]. The main result, on which our algorithm is based, is the following characterization of a degree sequence of a tree. 
Theorem 2.1. A sequence of integers $D=\left(d_{1}, d_{2}, \cdots, d_{n}\right)$, with $n-1 \geq d_{1} \geq d_{2} \geq$ $\cdots \geq d_{n-m}>d_{n-m+1}=\cdots=d_{n}=1$, is the degree sequence of a tree if and only if $C=\left(c_{1}, c_{2}, \cdots, c_{n-d_{n-m}+1}\right)$ is the degree sequence of a tree, where

$$
c_{i}= \begin{cases}d_{i} & i \leq n-m-1 \\ 1 & \text { otherwise }\end{cases}
$$

Proof. Let $T_{C}$ be a tree with degree sequence $C=\left(c_{1}, c_{2}, \cdots, c_{n-d_{n-m}+1}\right)$, with $c_{1} \geq c_{2} \geq$ $\cdots \geq c_{n-m-1}>c_{n-m}=\cdots=c_{n-d_{n-m}+1}=1$ and $c_{n-m-1} \geq d_{n-m} \geq 2$, satisfying (2).

To prove the easier direction of the equivalence, just add $d_{n-m}-1$ pendant vertices to a pendant vertex of $T_{C}$, obtaining a tree $T_{D}$. The degree sequence that corresponds to $T_{D}$ is $D=\left(d_{1}, d_{2}, \cdots, d_{n}\right)$, with $n-1 \geq d_{1} \geq d_{2} \geq \cdots \geq d_{n-m}>d_{n-m+1}=\cdots=d_{n}=1$.

The other direction of the equivalence, we prove as follows. Let $D=\left(d_{1}, d_{2}, \cdots, d_{n}\right)$, with $n-1 \geq d_{1} \geq d_{2} \geq \cdots \geq d_{n-m}>d_{n-m+1}=\cdots=d_{n}=1$, be a degree sequence of a tree $T_{D}$. Let $v_{n-m}$ be the vertex with degree $d_{n-m}$. If $v_{n-m}$ has $d_{n-m}-1$ pendant vertices, then delete them obtaining the tree $T_{C}$. If this is not a case, i.e., $v_{n-m}$ has $d>1$ adjacent vertices of degree bigger than one that comprised a set $U=\left\{u_{1}, u_{2}, \ldots, u_{d}\right\}$. Let $U_{1}$ be a set of adjacent vertices to $u_{1}$. First, delete all edges between $u_{1}$ and vertices in $U_{1} \backslash\left\{v_{n-m}\right\}$ and add edges between vertices in $U_{1} \backslash\left\{v_{n-m}\right\}$ and a pendant vertex whose distance to $u_{1}$ is bigger than its distance to any other vertex in $U$. Notice that $T_{D}$ has more than $d_{n-m}$ pendant vertices, therefore such pendant vertex must exists. Repeat the same as for $u_{1}$, for the rest of the vertices $u_{2}, u_{3}, \ldots, u_{d}$, considering one vertex per step until $v_{n-m}$ has $d_{n-m}-1$ pendant vertices, obtaining a tree $T_{D}^{\prime}$. Observe that $T_{D}^{\prime}$ has the same degree sequence as $T_{D}$. Finally, in $T_{D}^{\prime}$ delete all $d_{n-m}-1$ pendant vertices adjacent to $v_{n-m}$, obtaining the tree $T_{C}$.

Let $\mathbf{S}_{\mathbf{i}}$ be the set of all sequences $D_{i}=\left(d_{1}, d_{2}, \cdots, d_{i}\right)$, with fixed length $i$, where $1<i \leq n$ and $n-1 \geq d_{1} \geq d_{2} \geq \cdots \geq d_{h_{i}}>d_{h_{i}+1}=\cdots=d_{i}=1$. Notice that, $d_{h_{i}}$ denotes the smallest degree in $D_{i}$ larger than one. Define a function $f_{i}: \mathbf{S}_{\mathbf{i}} \times d_{h_{i}} \rightarrow \mathbf{S}_{\mathbf{i}-\mathbf{d}_{\mathbf{h}_{\mathbf{i}}}+\mathbf{1}} \times d_{h_{i}-1}$ such that for a given $D_{i} \in \mathbf{S}_{\mathbf{i}}$, and $C=\left(c_{1}, c_{2}, \cdots, c_{i-c_{h_{i}}+1}\right)$, it holds that $\left(C, c_{h_{i}-1}\right)=f_{i}\left(D_{i}, d_{h_{i}}\right)$ if

$$
c_{k}= \begin{cases}d_{k} & k \leq h_{i}-1 \\ 1 & \text { otherwise }\end{cases}
$$

By Theorem 2.1 and definition of the function $f_{i}$, we have the following two corollaries.

Corollary 2.2. For $i>0$ and $D_{i} \in \mathbf{S}_{\mathbf{i}}$, the sequence $D_{i} \in \mathbf{D}_{\mathbf{i}}$ if and only if $f_{i}\left(D_{i}, d_{h_{i}}\right)=$ $\left(D_{i-d_{h_{i}}+1}, d_{h_{i}-1}\right) \in \mathbf{D}_{\mathbf{i}-\mathbf{d}_{\mathbf{h}_{\mathbf{i}}}+\mathbf{1}}$.

Corollary 2.3. Let $C=\left(c_{1}, c_{2}, \ldots, c_{h_{i}} \ldots, c_{i-z}, c_{i-z+1}\right) \in \mathbf{D}_{\mathbf{i}-\mathbf{z}+\mathbf{1}}$, with $c_{h_{i}}$ the smallest degree bigger than 1 , and $2 \leq z \leq c_{h_{i}}$. The sequence $D_{i}=\left(d_{1}, d_{2}, \ldots, d_{i}\right) \in f_{i}^{-1}\left(C, c_{h_{i}}\right)$ if and only if

$$
d_{k}= \begin{cases}c_{k} & k \leq h_{i} \\ z & k=h_{i}+1 \\ 1 & \text { otherwise }\end{cases}
$$


The following example illustrates Corollary 2.3:

$$
f^{-1}(65111111111) \supseteq\{655111111111111,65411111111111,6531111111111,652111111111\} .
$$

One may straightforwardly implement Corollary 2.3 using recursion to enumerate degree sequences of trees. Our $\mathrm{C}++$ implementation was run on $2.3 \mathrm{GHz}$ Intel Core i5 processor with 4GB $1333 \mathrm{MHz}$ DDR3 RAM. The performance of the algorithm is presented in Table 1.

Table 1: Performance of the algorithm for enumerating degree sequences of trees. For degree sequences of length $n, S(n)$ denotes the number of degree sequences, $T(n)$ denotes the total running time and $S(n) / T(n)$ denotes the amortized running time for generating a sequence.

\begin{tabular}{||r|r|r|r|r|r|r|r||}
\hline \hline$n$ & $S(n)$ & $T(n)$ & $T(n) / S(n)[\mathrm{ms}]$ & $n$ & $S(n)$ & $T(n)$ & $T(n) / S(n)[\mathrm{ms}]$ \\
\hline \hline 21 & 490 & $3.031 \mathrm{~ms}$ & 0.00618571 & 34 & 8349 & $0.041 \mathrm{~s}$ & 0.00502455 \\
\hline 22 & 627 & $3.989 \mathrm{~ms}$ & 0.00636204 & 35 & 10143 & $0.051 \mathrm{~s}$ & 0.00501538 \\
\hline 23 & 792 & $4.875 \mathrm{~ms}$ & 0.00615530 & 40 & 26015 & $0.098 \mathrm{~s}$ & 0.00378359 \\
\hline 24 & 1002 & $6.082 \mathrm{~ms}$ & 0.00606986 & 50 & 147273 & $0.612 \mathrm{~s}$ & 0.00415907 \\
\hline 25 & 1255 & $7.884 \mathrm{~ms}$ & 0.00628207 & 60 & 715220 & $3.230 \mathrm{~s}$ & 0.00451627 \\
\hline 26 & 1575 & $9.996 \mathrm{~ms}$ & 0.00634667 & 70 & 3087735 & $15.300 \mathrm{~s}$ & 0.00495518 \\
\hline 27 & 1958 & $13.083 \mathrm{~ms}$ & 0.00668182 & 80 & 12132164 & $1 \mathrm{~m} 3 \mathrm{~s}$ & 0.00520250 \\
\hline 28 & 2436 & $14.434 \mathrm{~ms}$ & 0.00592529 & 90 & 44108109 & $4 \mathrm{~m} 5 \mathrm{~s}$ & 0.00556759 \\
\hline 29 & 3010 & $20.086 \mathrm{~ms}$ & 0.00667309 & 100 & 150198136 & $14 \mathrm{~m} 27 \mathrm{~s}$ & 0.00577865 \\
\hline 30 & 3718 & $18.821 \mathrm{~ms}$ & 0.00506213 & 110 & 483502844 & $51 \mathrm{~m} 26 \mathrm{~s}$ & 0.00635496 \\
\hline 31 & 4565 & $23.031 \mathrm{~ms}$ & 0.00504513 & 120 & 1482074143 & $2 \mathrm{~h} 39 \mathrm{~m} 8 \mathrm{~s}$ & 0.00647126 \\
\hline 32 & 5604 & $29.523 \mathrm{~ms}$ & 0.00526820 & 130 & 4351078600 & $7 \mathrm{~h} 36 \mathrm{~m} 43 \mathrm{~s}$ & 0.00629813 \\
\hline 33 & 6842 & $33.430 \mathrm{~ms}$ & 0.00488600 & 140 & 12292341831 & $21 \mathrm{~h} 7 \mathrm{~m} 44 \mathrm{~s}$ & 0.00618793 \\
\hline \hline
\end{tabular}

The time needed for generating a degree sequence indicates that algorithm runs in constant amortized time, so the total running time of the whole program is $O(S(n))$. Similarly as in the case of general graphs [41], it may be rather difficult to determine the precise complexity of the algorithm as function of $n$, which remains an open problem.

We have tested and analyzed our enumeration algorithm of degree sequences up to $n=$ 140, for which the algorithm ran about 21 hours. On our single processor platform, we would expect that for enumerating degree sequences of length 160 will run about 7 days. One can improve the computation by considering the distribution of the graphical sequences according to their first element. That can help to design an algorithm that computes the new values of $S(n)$ by slicing of the computations belonging to a given value of $n$, similarly as it was done in [37].

Having all degree sequences of a particular length, in the next section we proceed to determine the trees with minimal $\mathrm{ABC}$ index. 


\section{Trees with minimal atom-bond connectivity index}

Our algorithm of identifying the trees with minimal $\mathrm{ABC}$ index is comprised of the following steps:

1. Enumerate all degree sequences as described in Section 2.

2. Find corresponding 'greedy trees' for each generated degree sequence applying Theorem 1.3.

3. Calculate the ABC index of each 'greedy tree' and select the tree with minimal value.

Computationally, step 1. is the most expensive one, and as pointed in Section 2, it can be parallelized to some extend to run in distributed framework. Steps 2. and 3. can be implemented straightforwardly and there computational cost is linear with the respect to the length of the degree sequence. Although this approach is computationally superior to the computer assisted search presented in [25], it can be considerably improved by enumerating only the degree sequences that satisfy the following structural properties of the minimal $\mathrm{ABC}$ trees.

Proposition 3.1 ([32]). If $n \geq 10$, then the $n$-vertex tree with minimal $A B C$ index does not contain pendent paths of length $k \geq 4$.

Proposition 3.2 ([32]). If $n \geq 10$, then the $n$-vertex tree with minimal $A B C$ index contains at most one pendent path of length $k=3$.

Proposition 3.3 ([39]). If $n \geq 10$, then each pendent vertex of the $n$-vertex tree $G$ with minimal $A B C$ index belongs to a pendent path of length $k, 2 \leq k \leq 3$.

Considering all these results, that reduce significantly the number of degree sequences, we have implemented an algorithm that identifies trees with minimal $\mathrm{ABC}$ index. On our single processor platform, we have calculated all trees with minimal ABC index of order up to 300 in about 15 days, which is a significant improvement over the previous similar work [25], where a grid infrastructure of about 400 CPUs was used to identify all trees with minimal ABC index of order up to 31. All obtained trees with minimal ABC index are summarized in Figures 1 and 2. For the sake of completeness, we include also the results for $7 \leq n \leq 31$, which were already obtained in $[25,32]$. For $n \leq 6$, the minimal $\mathrm{ABC}$ trees are paths $P_{n}$ and they are omitted in the figures.

From the examples in Figures 1 and 2, the following observation can be made: If $\Delta_{1}$ is a maximal degree of a minimal ABC-tree with $n$ vertices, and $\Delta_{2}$ is a maximal degree of a minimal ABC-tree with $n+1$ vertices, then $\Delta_{2}=\Delta_{1} \pm 1$. Note that this observation is not necessarily true for larger minimal ABC-trees. However, applying the above restriction, one can significantly speed up the calculations. In this way we were able to obtain results that lead to disprovement of some existing conjectures. The plausible structural computational model and its refined version in [25], is based on the main assumption that the minimal ABC tree posses a single central vertex, or said with other words, it is based on the assumption 

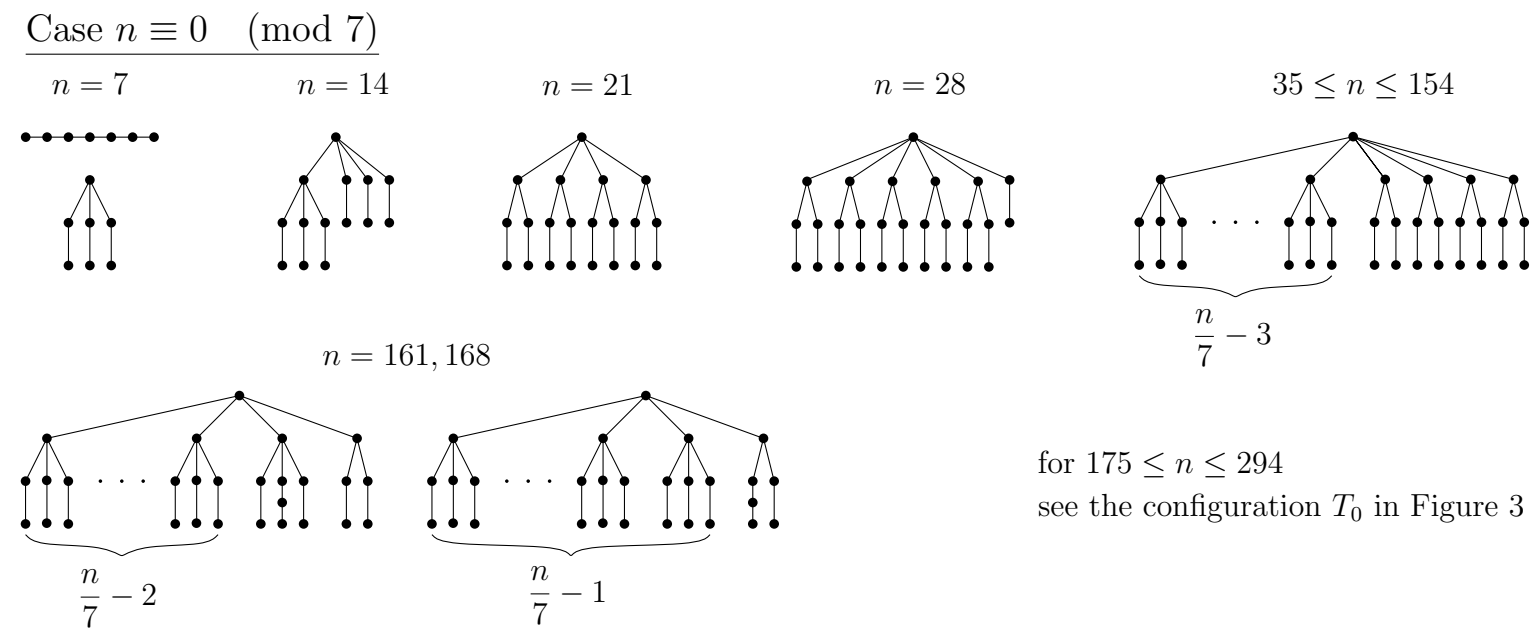

for $175 \leq n \leq 294$

see the configuration $T_{0}$ in Figure 3

Case $n \equiv 1 \quad(\bmod 7)$

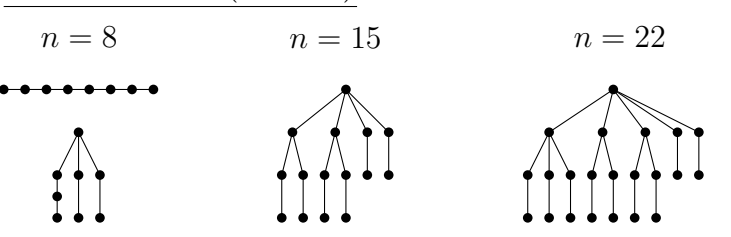

$n=43,50,57$

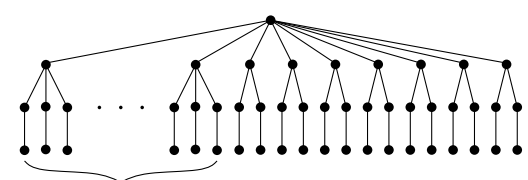

for $64 \leq n \leq 295$

see the configuration $T_{1}$ in Figure 3

$\left\lceil\frac{n}{7}\right\rceil-6$

Case $n \equiv 2 \quad(\bmod 7)$

$n=9$

$n=16$

$n=23$

$n=30$
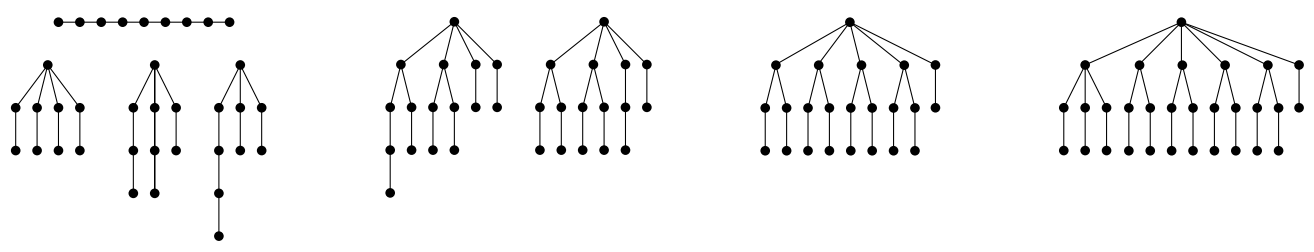

$n=37$

$44 \leq n \leq 163$

$170 \leq n \leq 296$
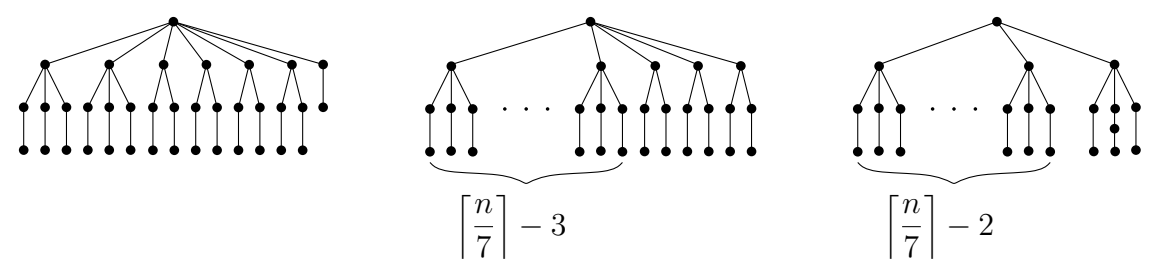

Figure 1: Trees of order $n, 7 \leq n \leq 300$, with minimal ABC index obtained by computer search - cases $n \equiv 0,1,2(\bmod 7)$. 
Case $n \equiv 3 \quad(\bmod 7)$

$n=10 \quad n=17$
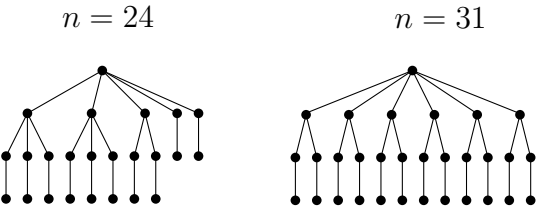

for $80 \leq n \leq 297$ see the configuration $T_{3}$ in Figure 3

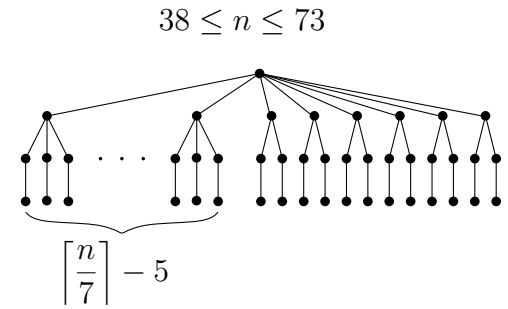

Case $n \equiv 4 \quad(\bmod 7)$
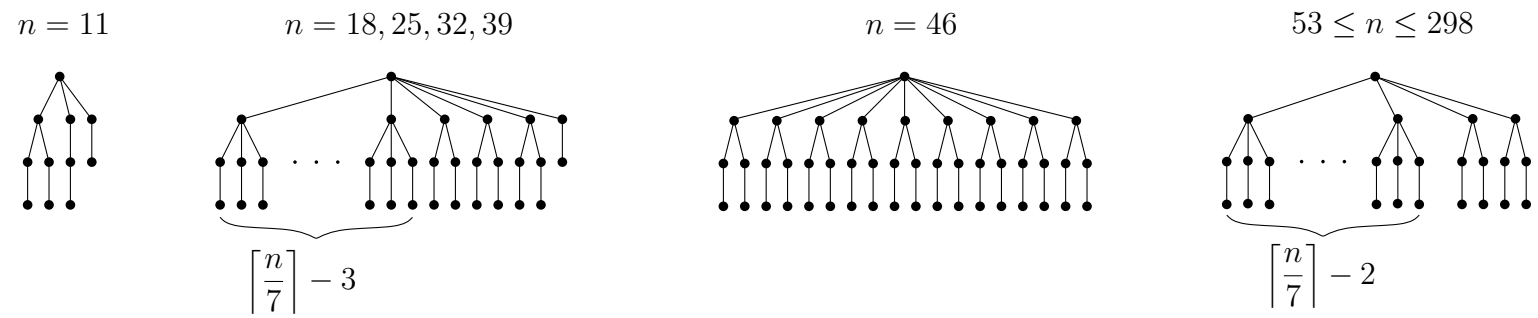

Case $n \equiv 5 \quad(\bmod 7)$

$n=12 \quad n=19$

$n=26$

$33 \leq n \leq 110$
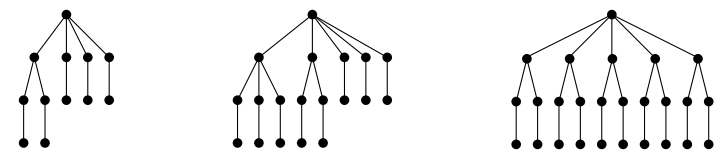

for $117 \leq n \leq 299$ see the configuration $T_{5}$ in Figure 3

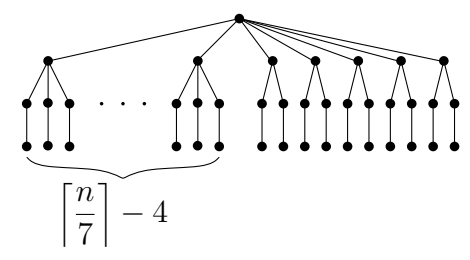

Case $n \equiv 6 \quad(\bmod 7)$

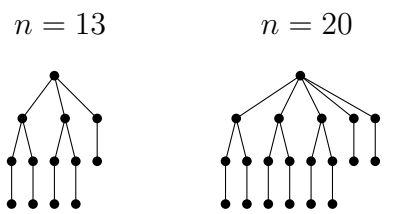

$n=55$

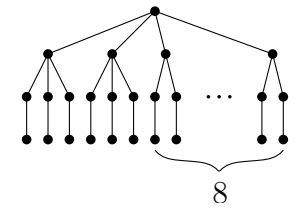

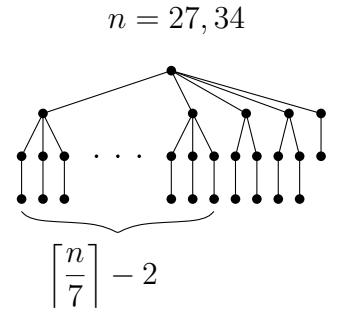
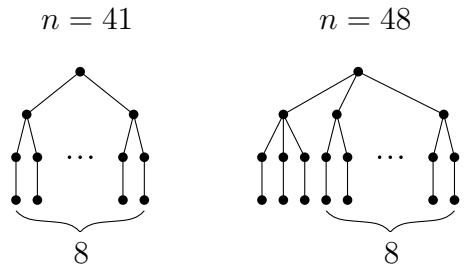

for $62 \leq n \leq 300$ see the configuration $T_{6}$ in Figure 3

Figure 2: Trees of order $n, 7 \leq n \leq 300$, with minimal ABC index obtained by computer search - cases $n \equiv 3,4,5,6(\bmod 7)$. 
that the vertices of a minimal $\mathrm{ABC}$ tree of degree $\geq 3$ induce a star graph. The configuration $T_{4}$ in Figure 3 , for $n \equiv 4(\bmod 7)$ and $n \geq 312$, is an counterexample to that assumption.

Applying the above constrain on the maximal degree of a minimal ABC-tree, we have also obtained another counterexample. Namely, the configuration $T_{2}$ in Figure 3 indicates different structure of the minimal ABC trees, in the case when $n \equiv 2(\bmod 7)$ and $n \geq 1185$, than the structure suggested for this case in $[25,30]$.

In meantime, independently to this work, the above two counterexamples were discovered and recently published. Namely, the counterexample $T_{4}$ in Figure 3 was published in [1], and the counterexample $T_{2}$ in Figure 3 was published in [2]. These two counterexamples, as well as the most already obtained results on this topic, will appear in a review article [31].

As a consequence to these counterexamples, we present a revised version of the conjecture by Gutman and Furtula [30] about the trees with minimal ABC index. Before we state it, it is worth to mention that the original versions of the conjecture by Gutman and Furtula, with slightly corrections, but still supporting the idea of existence of a central vertex, was shown to be true for the so-called Kragujevac trees [36].

Conjecture 3.1. Let $G$ be a tree with minimal $A B C$ index among all trees of size $n$.

(i) If $n \equiv 0(\bmod 7)$ and $n \geq 175$, then $G$ has the structure $T_{0}$ depicted in Figure 3.

(ii) If $n \equiv 1(\bmod 7)$ and $n \geq 64$, then $G$ has the structure $T_{1}$ depicted in Figure 3.

(iii) If $n \equiv 2(\bmod 7)$ and $n \geq 1185$, then $G$ has the structure $T_{2}$ depicted in Figure 3.

(iv) If $n \equiv 3(\bmod 7)$ and $n \geq 80$, then $G$ has the structure $T_{3}$ depicted in Figure 3.

(v) If $n \equiv 4(\bmod 7)$ and $n \geq 312$, then $G$ has the structure $T_{4}$ depicted in Figure 3.

(vi) If $n \equiv 5(\bmod 7)$ and $n \geq 117$, then $G$ has the structure $T_{5}$ depicted in Figure 3.

(vii) If $n \equiv 6(\bmod 7)$ and $n \geq 62$, then $G$ has the structure $T_{6}$ depicted in Figure 3.

The obtained computational results also indicate the following extension of the Proposition 3.2 .

Conjecture 3.2. A minimal $A B C$ tree of order $n>1178$ does not contain a pendant path of length three.

We would like to note that our computations show only minor violation of the assumption about the central vertex. However, to determine how big this violation is, is still an open problem. The computations here also strengthen the already existing believe, supported along by the computational model in [25], that the minimal $\mathrm{ABC}$ tree is unique for trees of order larger than 168.

\section{Acknowledgment}

The author thanks Boris Furtula for presenting the problem of characterizing graphs with minimal atom-bond connectivity index and sharing initial information about it. 
$T_{0}$

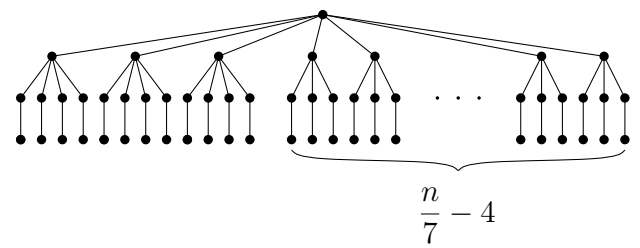

$T_{2}$

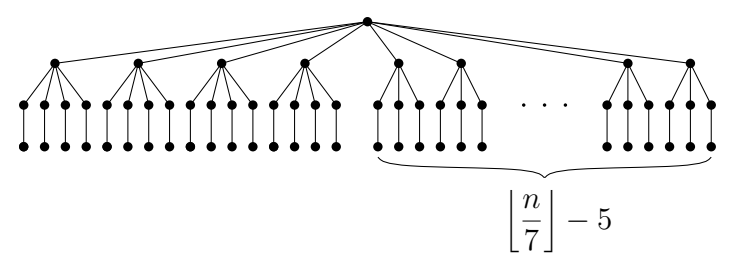

$T_{4}$

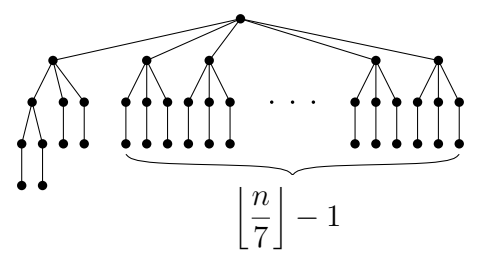

$T_{1}$

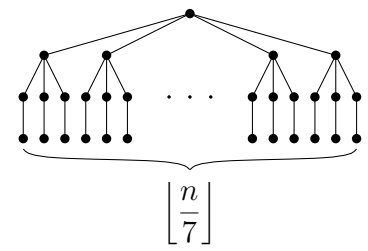

$T_{3}$

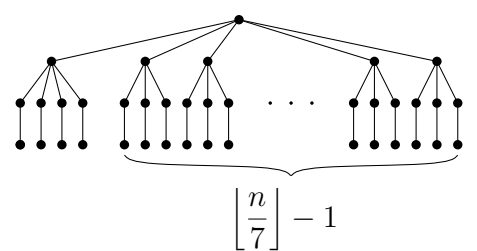

$T_{5}$

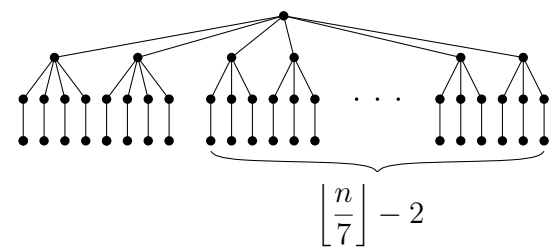

$T_{6}$

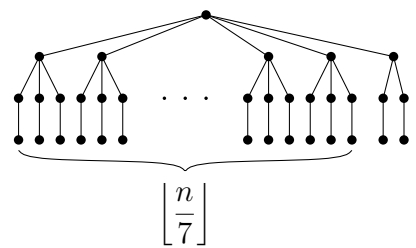

Figure 3: Types of trees with minimal ABC index that correspond to Conjecture 3.1.

\section{References}

[1] M. B. Ahmadi, S. A. Hosseini, P. Salehi Nowbandegani, On trees with minimal atom bond connectivity index, MATCH Commun. Math. Comput. Chem. 69 (2013) 559-563.

[2] M. B. Ahmadi, S. A. Hosseini, M. Zarrinderakht, On large trees with minimal atom-bond connectivity index, MATCH Commun. Math. Comput. Chem. 69 (2013) 565-569.

[3] M. B. Ahmadi, M. Sadeghimehr, Atom bond connectivity index of an infinite class $\mathrm{NS}_{1}[n]$ of dendrimer nanostars, Optoelectron. Adv. Mat. 4 (2010) 1040-1042.

[4] D. Avis, K. Fukuda, Reverse search for enumeration, Discrete Appl. Math. 2 (1996) $21-46$. 
[5] A. T. Balaban, Highly discriminating distance-based topological index, Chem. Phys. Lett. 89 (1982) 399-404.

[6] D. Bonchev, Information Theoretic Indices for Characterization of Chemical Structures, Research Studies Press, Chichester, 1983.

[7] J. Chen, X. Guo, Extreme atom-bond connectivity index of graphs, MATCH Commun. Math. Comput. Chem. 65 (2011) 713-722.

[8] J. Chen, X. Guo, The atom-bond connectivity index of chemical bicyclic graphs, Appl. Math. J. Chinese Univ. 27 (2012) 243-252.

[9] J. Chen, J. Liu, X. Guo, Some upper bounds for the atom-bond connectivity index of graphs, Appl. Math. Lett. 25 (2012) 1077-1081.

[10] J. Chen, J. Liu, Q. Li, The atom-bond connectivity index of catacondensed polyomino graphs, Discrete Dyn. Nat. Soc. 2013 (2013) ID 598517.

[11] K. C. Das, Atom-bond connectivity index of graphs, Discrete Appl. Math. 158 (2010) $1181-1188$.

[12] K. C. Das, I. Gutman, B. Furtula, On atom-bond connectivity index, Chem. Phys. Lett. 511 (2011) 452-454.

[13] K. C. Das, I. Gutman, B. Furtula, On atom-bond connectivity index, Filomat 26 (2012) $733-738$.

[14] K. C. Das, N. Trinajstić, Comparison between first geometric-arithmetic index and atom-bond connectivity index, Chem. Phys. Lett. 497 (2010) 149-151.

[15] M. Dehmer, M. Grabner, K. Varmuza, Information indices with high discriminative power for graphs, PLoS ONE 7 (2012) e31214.

[16] M. Dehmer, V. Kraus, On extremal properties of graph entropies, MATCH Commun. Math. Comput. Chem. 68 (2012) 889-912.

[17] M. Dehmer, A. Mowshowitz, A history of graph entropy measures, Inf. Sci. 181 (2011) $57-78$.

[18] J. Devillers, A T. Balaban (Eds.), Topological indices and related descriptors in QSAR and QSPR, Wiley-VCH, Gordon and Breach, Amsterdam, 1999.

[19] E. Estrada, Characterization of 3D molecular structure, Chem. Phys. Lett. 319 (2000) $713-718$.

[20] E. Estrada, Atom-bond connectivity and the energetic of branched alkanes, Chem. Phys. Lett. 463 (2008) 422-425.

[21] E. Estrada, L. Torres, L. Rodríguez, I. Gutman, An atom-bond connectivity index: Modelling the enthalpy of formation of alkanes, Indian J. Chem. 37A (1998) 849-855.

[22] G. H. Fath-Tabar, B Vaez-Zadeh, A. R. Ashrafi, A. Graovac, Some inequalities for the atom-bond connectivity index of graph operations, Discrete Appl. Math. 159 (2011) $1323-1330$.

[23] B. Furtula, A. Graovac, D. Vukičević, Atom-bond connectivity index of trees, Discrete Appl. Math. 157 (2009) 2828-2835. 
[24] B. Furtula, I. Gutman, M. Dehmer, On structure-sensitivity of degree-based topological indices, Appl. Math. Comput. 219 (2013) 8973-8978.

[25] B. Furtula, I. Gutman, M. Ivanović, D. Vukičević, Computer search for trees with minimal ABC index, Appl. Math. Comput. 219 (2012) 767-772.

[26] L. Gan, H. Hou, B. Liu, Some results on atombond connectivity index of graphs, MATCH Commun. Math. Comput. Chem. 66 (2011) 669-680.

[27] L. Gan, B. Liu, Z. You, The ABC index of trees with given degree sequence, MATCH Commun. Math. Comput. Chem. 68 (2012) 137-145.

[28] A. Graovac, M. Ghorbani, A new version of the atom-bond connectivity index, Acta Chim. Slov. 57 (2010) 609-612.

[29] I. Gutman, The energy of a graph, Ber. Math.-Statist. Sekt. Forschungsz. Graz 103 (1978) 1-22.

[30] I. Gutman, B. Furtula, Trees with smallest atom-bond connectivity index, MATCH Commun. Math. Comput. Chem. 68 (2012) 131-136.

[31] I. Gutman, B. Furtula, M. B. Ahmadi, S. A. Hosseini, P. Salehi Nowbandegani, M. Zarrinderakht, The ABC index conundrum, Filomat 27 (2013) 1075-1083.

[32] I. Gutman, B. Furtula, M. Ivanović, Notes on trees with minimal atom-bond connectivity index, MATCH Commun. Math. Comput. Chem. 67 (2012) 467-482.

[33] I. Gutman, J. Tošović, S. Radenković, S. Marković, On atom-bond connectivity index and its chemical applicability, Indian J. Chem. 51A (2012) 690-694.

[34] I. Gutman, N. Trinajstić, Graph Theory and Molecular Orbitals. Total $\pi$-electron Energy of Alternant Hydrocarbons, Chem. Phys. Lett. 17 (1971) 535-538.

[35] H. Hosoya, Topological index. A newly proposed quantity characterizing the topological nature of structural isomers of saturated hydrocarbons, Bull. Chem. Soc. Japan 44 (1971) $2332-2339$.

[36] S. A. Hosseini, M. B. Ahmadi, I. Gutman, Kragujevac trees with minimal atom-bond connectivity index, MATCH Commun. Math. Comput. Chem. 71 (2014) 5-20.

[37] A. Iványi, L. Lucz, T. F. Móri, P. Sótér, On Erdős-Gallai and Havel-Hakimi algorithms, Acta Univ. Sapientiae, Inform. 3 (2011) 230-268.

[38] X. Ke, Atombond connectivity index of benzenoid systems and fluoranthene congeners, Polycycl. Aromat. Comp. 32 (2012) 27-35.

[39] W. Lin, X. Lin, T. Gao, X. Wu, Proving a conjecture of Gutman concerning trees with minimal ABC index, MATCH Commun. Math. Comput. Chem. 69 (2013), 549-557.

[40] M. Randić, On characterization of molecular branching, J. Am. Chem. Soc. 97 (1975), 6609-6615.

[41] F. Ruskey, R. Cohen, P. Eades, A. Scott, Alley CATs in search of good homes, Congr. Numer. 102 (1994) 97-110.

[42] N. J. A. Sloane, Sequence A004251/M1250, The on-line encyclopedia of integer sequences. 
[43] N. J. A. Sloane, Sequence A000055, The on-line encyclopedia of integer sequences.

[44] R. Todeschini, V. Consonni, Molecular descriptors for chemoinformatics, Wiley-VCH, Weinheim, 2009.

[45] T. S. Vassilev, L. J. Huntington, On the minimum ABC index of chemical trees, Appl. Math. 2 (2012) 8-16.

[46] H. Wang, Extremal trees with given degree sequence for the Randić index, Discrete Math. 308 (2008) 3407-3411.

[47] H. Wiener, Relation of the physical properties of the isomeric alkanes to molecular structure. Surface tension, specific dispersion, and critical solution temperature in aniline, J. Phys. Chem. 52 (1948) 1082-1089.

[48] R. Xing, B. Zhou, Extremal trees with fixed degree sequence for atom-bond connectivity index, Filomat 26 (2012) 683-688.

[49] R. Xing, B. Zhou, F. Dong, On atom-bond connectivity index of connected graphs, Discrete Appl. Math. 159 (2011) 1617-1630.

[50] R. Xing, B. Zhou, Z. Du, Further results on atom-bond connectivity index of trees, Discrete Appl. Math. 158 (2011) 1536-1545.

[51] J. Yang, F. Xia, H. Cheng, The atom-bond connectivity index of benzenoid systems and phenylenes, Int. Math. Forum 6 (2011) 2001-2005. 